\title{
A decision making algorithm for rehabilitation after stroke: A guide to choose an appropriate and safe treadmill training
}

\author{
Fabio Vanoglio $^{\mathrm{a}}$, Adriana Olivares ${ }^{\mathrm{b}, *}$, Gian Pietro Bonometti ${ }^{\mathrm{a}}$, Silvia Damiani ${ }^{\mathrm{a}}$, Marta Gaiani ${ }^{\mathrm{a}}$, \\ Laura Comini $^{\mathrm{b}}$ and Alberto Luisa ${ }^{\mathrm{a}}$ \\ ${ }^{a}$ Istituti Clinici Scientifici Maugeri IRCCS, Neurological Rehabilitation Unit of the Institute of Lumezzane, \\ Brescia, Italy \\ ${ }^{\mathrm{b}}$ Istituti Clinici Scientifici Maugeri IRCCS, Scientific Direction of the Institute of Lumezzane, Brescia, Italy
}

Received 23 February 2021

Accepted 21 April 2021

\begin{abstract}
.
BACKGROUND: Walking independently after a stroke can be difficult or impossible, and walking reeducation is vital. But the approach used is often arbitrary, relying on the devices available and subjective evaluations by the doctor/physiotherapist. Objective decision making tools could be useful.

OBJECTIVES: To develop a decision making algorithm able to select for post-stroke patients, based on their motor skills, an appropriate mode of treadmill training (TT), including type of physiotherapist support/supervision required and safety conditions necessary.

METHODS: We retrospectively analyzed data from 97 post-stroke inpatients admitted to a NeuroRehabilitation unit. Patients attended TT with body weight support (BWSTT group) or without support (FreeTT group), depending on clinical judgment. Patients' sociodemographic and clinical characteristics, including the Cumulative Illness Rating Scale (CIRS) plus measures of walking ability (Functional Ambulation Classification [FAC], total Functional Independence Measure [FIM] and Tinetti Performance-Oriented Mobility Assessment [Tinetti]) and fall risk profile (Morse and Stratify) were retrieved from institutional database.

RESULTS: No significant differences emerged between the two groups regarding sociodemographic and clinical characteristics. Regarding walking ability, FAC, total FIM and its Motor component and the Tinetti scale differed significantly between groups (for all, $p<0.001$ ). FAC and Tinetti scores were used to elaborate a decision making algorithm classifying patients into 4 risk/safety (RS) classes. As expected, a strong association (Pearson chi-squared, $p<0.0001$ ) was found between RS classes and the initial BWSTT/FreeTT classification.

CONCLUSION: This decision making algorithm provides an objective tool to direct post-stroke patients, on admission to the rehabilitation facility, to the most appropriate form of TT.
\end{abstract}

Keywords: Algorithm, body weight support, rehabilitation, safety, treadmill

\section{Introduction}

Stroke is one of the most common disabling conditions worldwide and the third most frequent cause of

*Address for correspondence: Adriana Olivares, Istituti Clinici Scientifici Maugeri IRCCS, Via Salvatore Maugeri. 4, 27100 Pavia, Italy. Tel.: +39 30 8253281; E-mail: adriana.olivares@ icsmaugeri.it. death (Gresham et al., 1979). Between 55\% and 75\% of post-stroke patients are characterized by severe motor function deficits, negatively affecting their quality of life and professional or daily life activities (Dionisio, Duarte, Patricio, \& Castelo-Branco, 2018). Difficulty or inability to walk is one of the most common effects of stroke, and restoration of gait is a primary goal of rehabilitation (Bohannon, Andrews, \& Smith, 1988). 
Recovery of walking ability can be achieved through a variety of rehabilitation approaches: 1) mechanically-assisted walking (MAW); 2) treadmill training (TT), eventually with body weight support (BWSTT); and 3) walking training on the ground (WTG). All these activities consist of exercises with different characteristics of intensity, repetitiveness and functionality (mobility task training) recommended (class I, level A) by the American Heart Association (AHA) 2016 guidelines (Winstein et al., 2016). According to these guidelines, different modes of gait training combined with conventional rehabilitation can be used for the recovery of walking function (class IIb, level A). In particular, BWSTT should be used in post-stroke patients who cannot walk independently or have limited capacity (class IIb, level A), with benefit especially in older subjects, with a greater gait impairment (Barbeau \& Visintin, 2003). The 2015 Canadian Guidelines (Casaubon et al., 2016) also recommend use of TT/BWSTT when WTG may not be feasible or safe. According to a recent review (Mehrholz, Thomas, \& Elsner, 2017), people who perform TT after stroke are not more likely to improve their walking ability than those who do not perform TT. Conventional rehabilitation exercise and WTG to date are at least as effective as more complex strategies such as treadmill or robot-based interventions (Dickstein, 2008). In agreement with other authors, Rensink et al. (Rensink, Schuurmans, Lindeman, \& Hafsteinsdottir, 2009), comparing three high-quality studies on the effects of WTG vs. TT performed in subacute and chronic phases, showed that both strategies improve performance without significant differences. Recently, some studies focused their attention on comparing different types of roboticassisted BWSTT or locomotor with standard BWSTT (Esquenazi et al., 2017) and on predictors, clinical outcomes, timing and type of walking rehabilitative interventions (Selves, Stoquart, \& Lejeune, 2020): no significant differences in efficacy, but different indications on timing between modalities, were underlined.

While there is no significant difference in efficacy between the methods, they can differ in terms of their practicability and safety. Therefore, based on the clinical assessment, the choice of the most appropriate rehabilitative method should be guided by a pragmatic approach (Dickstein, 2008) considering two fundamental points: the need for an appropriate selection of patients (with clear indications of "who" and "on what basis") (Ada, Dean, Hall, Bampton, \& Crompton, 2003; da Cunha et al., 2002; Franceschini et al., 2009; Gama et al., 2017; Ng, Tong, \& Li, 2008; Pang, Charlesworth, Lau, \& Chung, 2013) and the need to ensure the most appropriate safety conditions (Eng \& Tang, 2007; Mehrholz et al., 2017).

At present, however, the walking reeducation approach used depends on the number and type of devices available, recommendations from the literature ("White Book on Physical and Rehabilitation Medicine in Europe. Introductions, Executive Summary, and Methodology," 2018), and the doctor's clinical judgment and physiotherapist' evaluations. Specific decision making tools are few or lacking altogether. Considering the limited resources available in the health sector and also the more widespread diffusion of equipment such as MAW, TT, and TTBWS, it is essential to offer a service that, starting from the functional assessment of the patient and respecting efficiency and safety criteria, can enhance the effectiveness of the rehabilitation process without increasing the burden on the physiotherapist (whose role ideally should be, where possible, to guide the exercise program in a supervisory capacity).

The main purpose of this study was to identify, in post-stroke patients, the motor skills sufficient to permit TT use under the supervision of the physiotherapist in the most appropriate safety conditions. Based on these measured skills, we elaborated a decision making algorithm, permitting an objective, repeatable and safe decision on "if and how" to use TT as a walking reeducation intervention. A secondary aim was to evaluate if there were any adverse events or annotations relating to the use of TT in this type of patient.

\section{Material and methods}

\subsection{Study design}

The protocol was approved by the Ethical Committee of the Istituti Clinici Scientifici (ICS) Maugeri, Pavia, Italy (CEC 234010 September 2019). All patients provided informed consent for the scientific use of their data.

This was a one-time retrospective observational study performed on a cohort of patients whose data were in our institutional database, following STROBE reporting guidelines.

Patients with a diagnosis of stroke (subacute or chronic stroke) known at the neurological visit, and admitted to our NeuroRehabilitation unit in the period July 2018-December 2019 for a usual rehabilitation 
period of about two months, were considered. They were adults of both sexes to whom reeducation of walking was planned. Rehabilitation plan included TT with a comfortable speed and daily session of 10-15 minutes 5 out 7 days/week. During the usual rehabilitation, the physiotherapist (PT) tailored on the specific patient ability and needs the mode of TT with the safety condition (fall retention and/or double support) retained most appropriate based on clinical judgment and expertise (Barbeau \& Visintin, 2003). Clinical data and functional performance were evaluated for all patients (able, unable or at high risk for TT) following ICS Maugeri internal fall risk assessment (IO ICSM CGA rev0, 2018) and were collected in the institutional database.

\subsection{Algorithm development}

Information regarding sociodemographic characteristics, classification of stroke (subacute or chronic), presence of comorbidities (evaluated by Cumulative Illness Rating Scale (CIRS) (Parmelee, Thuras, Katz, \& Lawton, 1995), clinical scales of demonstrated reliability administered by the physiotherapist the day after admission and any adverse events/annotations registered during the rehabilitation process, were extracted from institutional database.

In the phase of the algorithm setup, data from patients who were considered by the physiotherapist to be unable to understand verbal instructions or motor commands or to have a high safety risk for TT because i) needing for total support in walking (Functional Ambulation Classification score $=0$ ) or ii) were in unstable clinical conditions (presence of uncontrolled epilepsy, apraxia, orthostatic hypotension, severe cognitive deficits) were excluded (Fig. 1).

For the development of the algorithm we considered clinical scales related to motor and balance skills as: the Functional Ambulation Classification (FAC) (Holden, Gill, \& Magliozzi, 1986) to assess the amount of human assistance required with walking; the Functional Independence Measure (FIM) (Granger, Cotter, Hamilton, \& Fiedler, 1993) to assess the patient's degree of independence and need of assistance in performing basic ADLs (motor and a cognitive subscales); the Tinetti Performance-Oriented Mobility Assessment (Tinetti, 1986) to assess patients' balance from a clinical point of view with (balance and gait

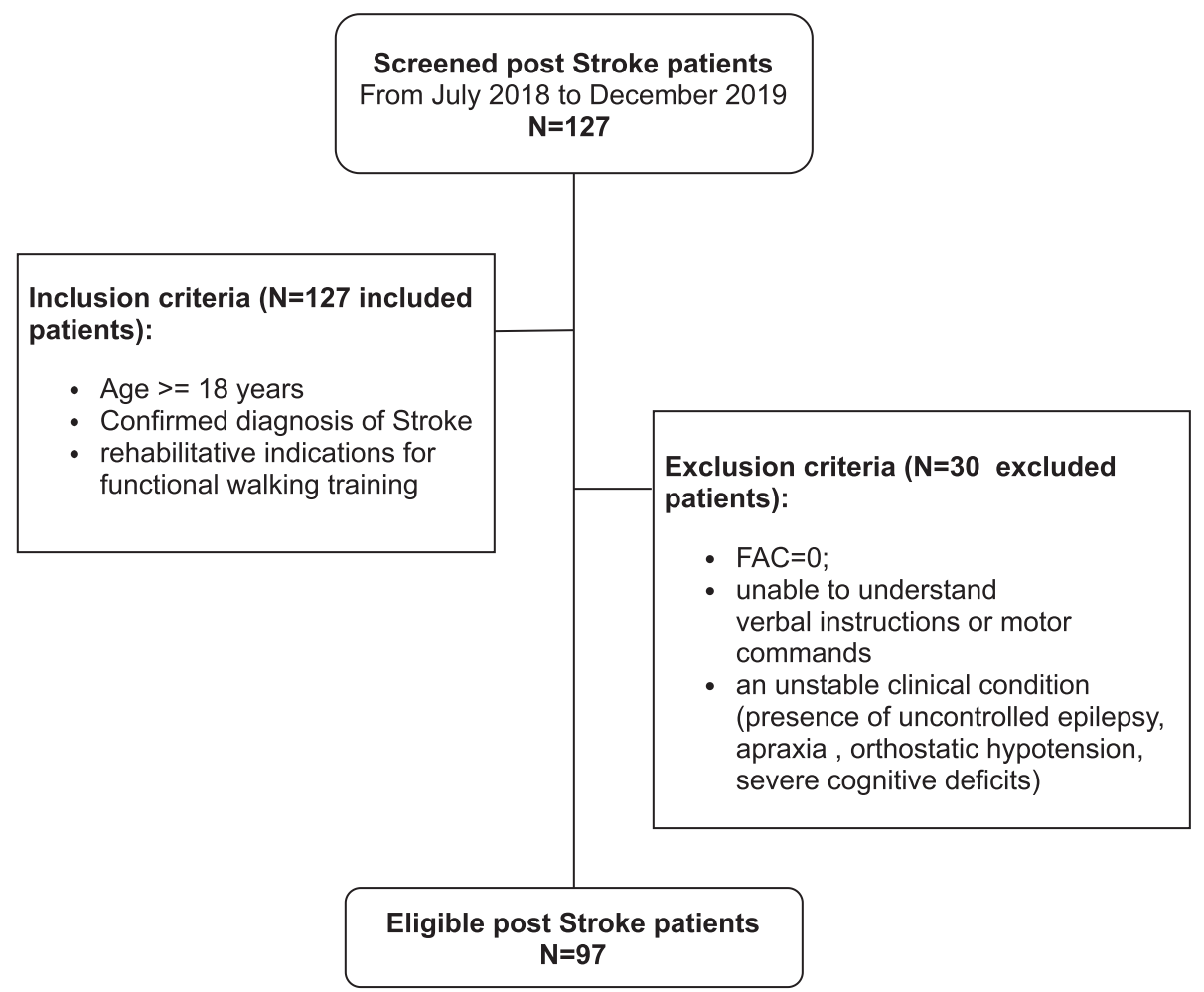

Fig. 1. Study flowchart. 
components); the Morse Fall Scale (MFS) (Morse, Morse, \& Tylko, 1989) and the Stratify scale (Oliver, Britton, Seed, Martin, \& Hopper, 1997) to investigate and quantify patients'risk of falling (see Supplementary Material for details).

All these evaluations performed in "patients able to perform TT with or without body weight support" were allocated in 2 groups as follows:

- BWSTT group = including patients who required body weight support to perform TT exercises

- FreeTT group = including patients who could perform TT exercises without the need for body weight support (totally free or using bars for support).

Comparison between groups on all scale scores was performed and, only in case of statistically significant difference, we established a cut-off value to identify different classes of risk in TT used. Cut off value was expressed as media $\pm 1 \mathrm{SD}$ or $1 \mathrm{st} / 3$ rd quartile according to parametric or not parametric nature of data. Adverse events were collected as well and reported to better define the applicability criteria of the algorithm and/or to assign patients directly to the higher security class.

Possible differences between clinical judgment and algorithm application were also evaluated in the two groups.

\subsection{Statistical analysis}

\subsubsection{Sample size estimate}

As this is an observational cohort study with retrospective evaluation, we did not carry out an estimate of the representative sample size. The number of patients recruited was conditioned by the available patients within the selected time period.

\subsubsection{Comparison between groups}

All data were evaluated for Gaussian distribution (Shapiro-Wilk test) before applying statistical analysis using the $\mathrm{R}$ programming language(24). Based on the result, Student's $t$-test or Wilcoxon test was applied to compare mean or median values between the BWSTT and FreeTT groups.

\subsubsection{Comparison between clinical judgment and algorithm application}

For frequency comparison analysis between groups from PT clinical judgment (FreeTT and BWSTT) and risk classes from the algorithm, we used Pearson chi-squared test with Monte Carlo correction in the case of low numbers.

For all analyses, a $p$ value $<0.05$ was considered statistically significant.

\section{Results}

One hundred twenty-seven post-stroke patients were evaluated at our facility in the period July 2018-December 2019. All of them met the inclusion criteria, but 30 patients were excluded because they were considered by the physiotherapist to be unable or at high safety risk for TT. The statistical analysis was therefore performed on retrospective data from 97 patients able to perform TT according to clinical indication (Fig. 1).

Table 1

Baseline demographic and clinical characteristics for the whole study population and BWSTT vs. FreeTT groups

\begin{tabular}{lcccc}
\hline & All & $\begin{array}{c}\text { BWSTT } \\
(n=24)\end{array}$ & $\begin{array}{c}\text { FreTT } \\
(n=73)\end{array}$ & $\begin{array}{c}P \\
(\text { delta; } 95 \% \mathrm{CI})\end{array}$ \\
\hline Sex, M/F & $47 / 50$ & $9 / 15$ & $38 / 35$ & 0.2463 \\
Age, years* & $75(68-80)$ & $77(69-85)$ & $75(68-80)$ & 0.1765 \\
Subacute/chronic Stroke & $56 / 41$ & $14 / 10$ & $42 / 31$ & 1.0000 \\
CIRS 1* & $2.0(1.7-2.4)$ & $1.9(1.8-2.5)$ & $2.0(1.7-2.4)$ & 0.5584 \\
CIRS 2* & $5.0(3.0-8.0)$ & $5.0(3.0-8.0)$ & $5.0(3.0-8.0)$ & 0.7461 \\
Total FIM & $83.39(21.59)$ & $70.38(17.96)$ & $87.67(21.05)$ & $\mathbf{0 . 0 0 0 3}$ \\
& & & & $(17.30 ; 8.40-26.19)$ \\
Cognitive FIM* & $30.0(24.0-34.0)$ & $27.0(24.0-33.5)$ & $31.0(35.0-34.0)$ & 0.3918 \\
MFS* $_{\text {Stratify* }}$ & $40.0(25.0-60.0)$ & $40.0(28.8-61.2)$ & $40.0(25.0-60.0)$ & 0.7518 \\
\hline
\end{tabular}

Legend: BWSTT = Body Weight Support Treadmill Training; FreeTT = Free Treadmill Training; CIRS = Cumulative Illness Rating Scale; FIM = Functional Independence Measure; FAC = Functional Ambulation Classification; MFS = Morse Fall scale; ${ }^{*}$ Median $(1 \mathrm{st}-3 \mathrm{rd}$ quartile $)$ and related Wilcoxon test; ${ }^{\wedge}$ Mean (Standard deviation) and related T Student test. 
A

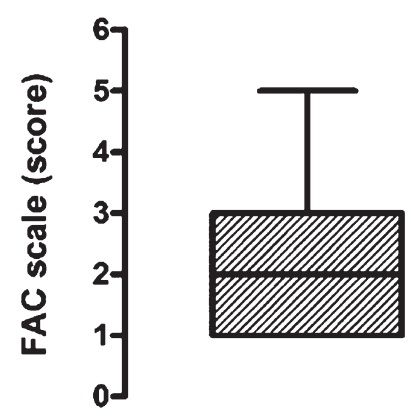

BWSTT
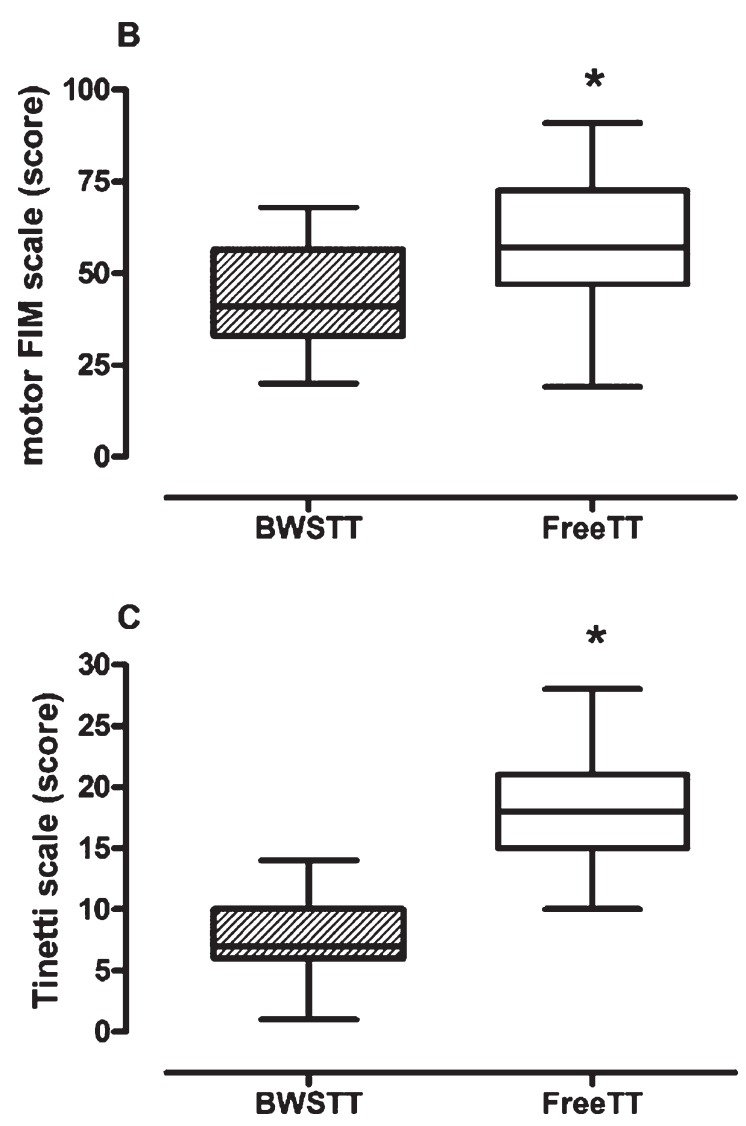

Fig. 2. FAC (panel A), Motor FIM (panel B) and Tinetti (panel C) Boxplot of BWSTT vs. FreeTT group. For the three scales higher score values indicate higher functioning. Legend:FreeTT $=$ Free treadmill training; BWSTT $=$ Body weight support treadmill training; FAC =Functional Ambulation Classification; FIM =Functional Independence Measure; Tinetti $=$ Tinetti Performance-Oriented Mobility Assessment $\mathrm{A}+\mathrm{B}$ component. ${ }^{*} p<0.0001$.

\subsection{Comparison between groups}

Table 1 and Fig. 2 show the demographic and clinical functional characteristics in the total population and in BWSTT and FreeTT groups.

The study population was composed of elderly individuals, admitted to rehabilitation after subacute $(58 \%)$ and chronic stroke (42\%), evenly divided between males ( $48 \%$ ) and females, with a low number of comorbidities (Table 1). No significant difference emerged regarding the sociodemographic and clinical characteristics between the two groups. On the contrary, functional characteristics as walking ability and balance (measured by the FAC, total FIM, MotorFIM, and Tinetti scale) differed significantly between the two groups (for all, $p<0.001$ ).

Figure 2 shows the boxplot distributions related to the three scales with significant differences between groups. Delta changes between groups were 2.00 for FAC $(95 \% \mathrm{CI}=1.00-2.00), 15.79$ for Motor FIM $(95 \% \mathrm{CI}=8.51-23.06)$ and 10.33 for Tinetti $(95 \% \mathrm{CI}=8.80-11.87)$.

These three scales were considered for the decision making algorithm development. The FAC 1st quartile value of the BWSTT group was the most important discriminating item and was used firstly in the algorithm to define patients with poor walking ability, particularly those for whom the use of TT should be in conditions of maximum safety (with the support of a dedicated operator).

The Tinetti Scale was the second discriminating item, useful to differentiate between BWSTT and FreeTT and to decide, in FreeTT, whether support on the bars is necessary or not. Motor-FIM was not included in the algorithm development. The Tinetti scale was preferred to the Motor-FIM because it enabled a better BWSTT/FreeTT stratification: the related Tinetti boxplots do not overlap (Fig. 2), with a greater significance and a narrower confidence interval.

\subsection{Algorithm development}

The FAC and Tinetti scales were included in the decision making algorithm (Fig. 3) that assigns patients to four risk/safety (RS) classes, as described below:

- RS class 4 (HIGH RISK/MAXIMUM SAFETY required): patients performing TT with need for both body weight support and further support/help of a dedicated physiotherapist (BWSTT clinical-assignment group); 


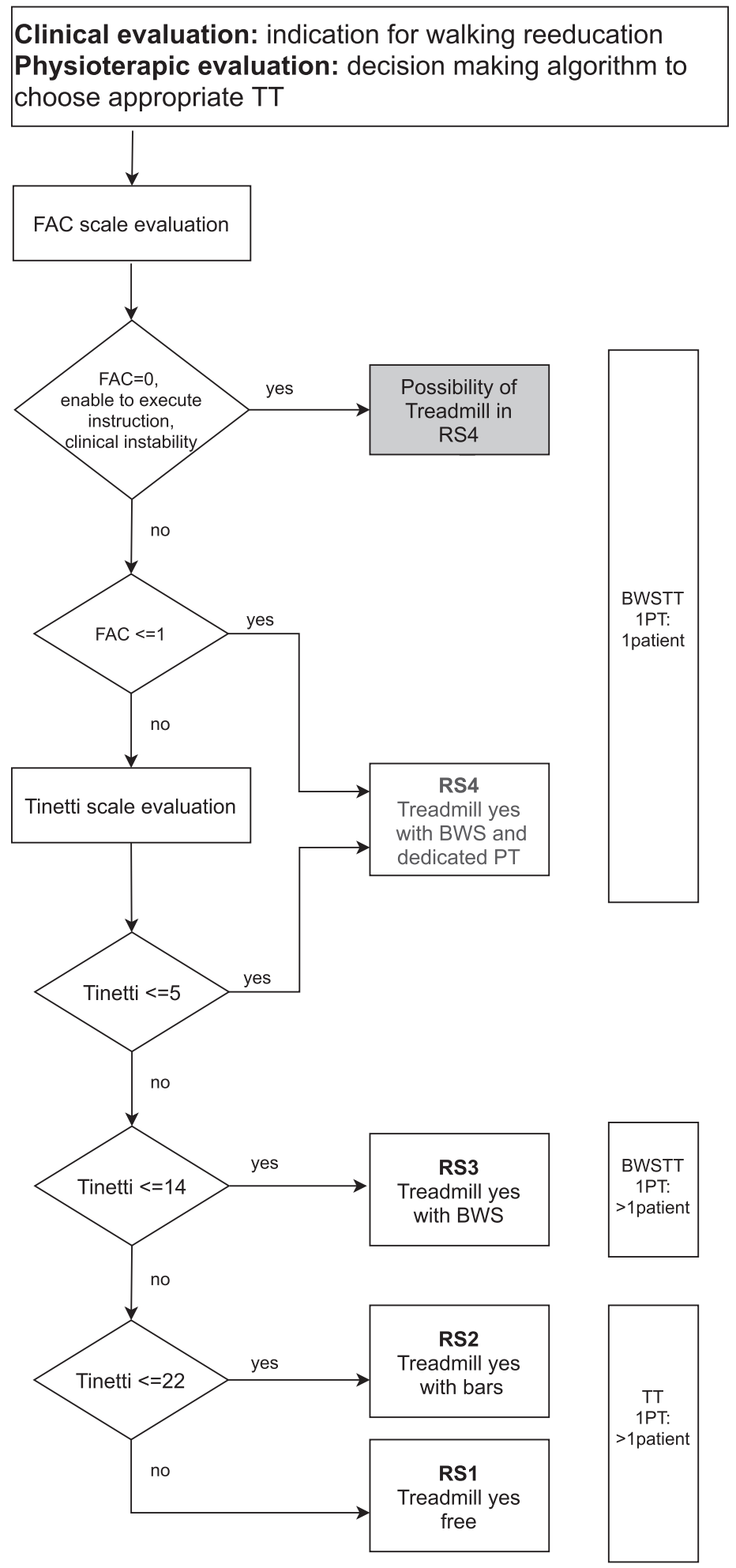

Fig. 3. Flowchart of Decision making algorithm for TT. Legend: FreeTT $=$ Free treadmill training; BWSTT = Body weight support treadmill training; FAC = Functional Ambulation Classification; FIM = Functional Independence Measure; Tinetti = Tinetti Performance-Oriented Mobility Assessment A + B component; PT = physiotherapist. 
- RS class 3: patients performing TT with body weight support and physiotherapist supervision (BWSTT clinical-assignment group);

- RS class 2: patients performing TT without body weight support but with the aid of bars and physiotherapist supervision (FreeTT clinicalassignment group);

- RS class 1: (LOW RISK/LOW SAFETY necessity): patients performing TT without body weight support or the aid of bars, but only under physiotherapist supervision (FreeTT clinicalassignment group).

The assignment to the risk class was based on the following considerations (Fig. 3):

1) For patients with $\mathrm{FAC} \leq 1$ (where 1 was $1 \mathrm{st}$ quartile value of the BWSTT group), the Tinetti scale value was not necessary (Tinetti A could be considered as an indicator of balance) and patients were directly assigned to RS class 4 . To the same risk class, with a careful and tailored clinical evaluation by PT, could be assigned also patients with $\mathrm{FAC}=0$ and/or inability to execute commands and/or with pathologies involving a high risk of falling (uncontrolled epilepsy, orthostatic hypotension, apraxia, severe cognitive deficits), initially excluded from TT.

2) For patients with FAC $>1$, the Tinetti scale value was used to further define the risk class:

- RS class 4 = for patients needing BWS with further support of a dedicated physiotherapist: Tinetti value $\leq 4.77$ (BWSTT mean - 1SD);

- RS class $3=$ for patients needing BWS: Tinetti value $>4.77$ and $\leq 13.85$ (FreeTT mean - 1SD)

- RS class $2=$ for patients not needing BWS but with the aid of bars: Tinetti value $>13.85$ and $\leq 22.15$ (FreeTT mean + 1SD)

- RS class $1=$ for FreeTT patients: Tinetti value $>22.15$

Therefore, using approximation to guarantee greater safety, discriminating cut-offs with or without health staff employment were confirmed in Figure 3 (Supplementary Materials for details).

\subsection{Comparison between clinical judgment and algorithm application}

The RS class for addressing BWS or Free Treadmill Treatment assigned by the decision making algorithm (dmA) was then compared with the initial clinical judgment (Fig. 4).

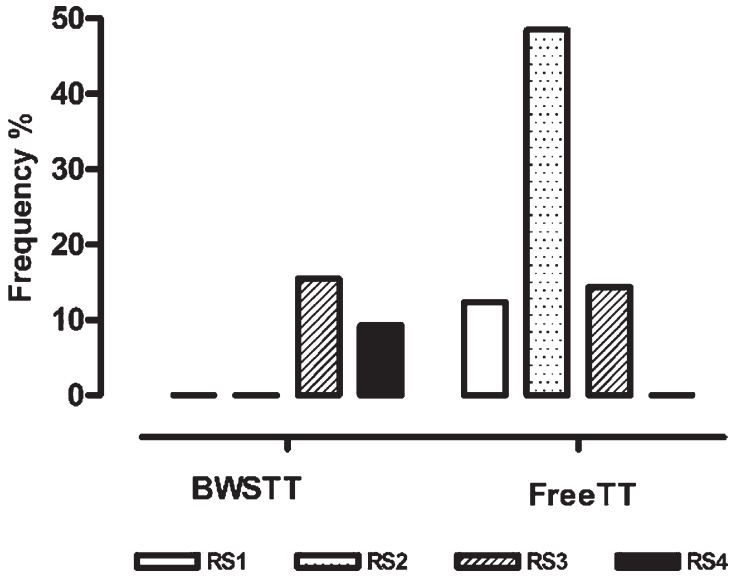

Fig. 4. RS classes from Decision making algorithm vs FreeTT/BWSTT initial groups. Legend: RS classes = Risk-safety classes; FreeTT $=$ free treadmill training; BWSTT $=$ Body weight supported treadmill training.

As expected, we found a strong association (Pearson chi-squared, $p<0.0001$ ), with $100 \%$ agreement between RS3 + RS4 evaluated by dmA and BWSTT clinical assignment (24/24 patients) and $81 \%$ agreement between RS1 + RS2 evaluated by $\mathrm{dmA}$ and FreeTT (59/73 patients). The cumulative percentage of agreement was $86 \%$. Discrepancies were found in $14 / 97$ patients (14\%), all considered as belonging to the initial FreeTT group but addressed by the algorithm to RS3 (BWS with PT supervision only), which enhances the condition of safe TT.

Adverse events were recorded in two patients (angina episode and fall), while four were firstly assigned at the lowest risk condition and then switched to a safer one by PT. See Supplementary Material for details.

\section{Discussion}

Our study has highlighted in post-stroke patients a significant difference in the FAC and Tinetti functional scores between patients who perform treadmill training with vs. without the need for body weight support. These scales emerged as the most useful scores to include in an algorithm to determine objectively "if and how" treadmill training can be used as a safe walking rehabilitation intervention in post-stroke patients.

Several studies exist on treadmill training in poststroke patients (Bohannon et al., 1988; Mehrholz et al., 2017). The characteristics of patients who can 
benefit or not from TT and under which specific safety conditions have been reported in papers and guidelines (Casaubon et al., 2016; Winstein et al., 2016).

Primarily, our aim here was to identify an algorithm able to indicate which patients can be "candidate" for TT. All patients with the following characteristics were a priori excluded by our algorithm: 1) presence of comorbidities that constitute a contraindication to physical exercise such as: cardiovascular diseases (Brauer, Kuys, Paratz, \& Ada, 2018; Gama et al., 2017; $\mathrm{Ng}$ et al., 2008); uncontrolled hypertension with values $>190 / 110 \mathrm{mmHg}$ (da Cunha et al., 2002); New York Heart Association classes III and IV (Franceschini et al., 2009; Globas et al., 2012); $\mathrm{C}$ and $\mathrm{D}$ risk class for exercise according to the American College of Sports Medicine (Franceschini et al., 2009); musculoskeletal problems (Bonnyaud et al., 2013; da Cunha et al., 2002); uncontrolled diabetes(da Cunha et al., 2002); severe cognitive deficits defined by Mini-Mental State Examination (MMSE) score $\leq 23$ (Luft et al., 2008) or MMSE $<27$ (Mao et al., 2015) or MMSE $\leq 21$ ( $\mathrm{Ng}$ et al., 2008); 2) language deficiencies that preclude the execution of orders (Ada, Dean, \& Lindley, 2013; Ada, Dean, Morris, Simpson, \& Katrak, 2010) or of two different commands at a time (Luft et al., 2008; $\mathrm{Ng}$ et al., 2008); 3 ) inability to walk independently ranging from a generic inability (Ada et al., 2003; Bonnyaud et al., 2013; Franceschini et al., 2009) to inability for at least $30 \mathrm{~m}$ (Kim, Gong, \& Kim, 2011) or for at least $3 \mathrm{~min}$ at $\geq 0.3 \mathrm{Km} / \mathrm{h}$ speed (Globas et al., 2012). According to these indications, we defined specific exclusion criteria for the current study.

Some authors (Pang et al., 2013) have drawn up a series of recommendations on prescription for TT, suggesting a division into high and low-risk patients, in particular according to acute or chronic phase and allocating training in a clinical setting or in the community/home, respectively. In our study, all patients were attending an in-hospital rehabilitative program.

Secondly, basing on data from selected patients, our decision making algorithm was developed to clearly identify the need for BWSTT for each patient. To this end, we used the FAC as the primary scale. In addition, we used the Tinetti scales to determine the need for an active presence of the physical therapist during TT.

TT, with or without BWS, is a rehabilitative option that allows patients to perform walking training early and in a safe condition (Eng \& Tang, 2007). But it is necessary to clearly state what level of walking ability is needed and when the use of the treadmill support bars may be sufficient to guarantee an adequate security. BWSTT is used in post-stroke patients who are not able to walk independently or have a limited capacity (Mehrholz et al., 2017), but again it is necessary to state functional ability required and the TT modality (with active presence of the physiotherapist or only supervision). Some criteria have been set for TT without support: from FAC with a score $>3$ (Kosak \& Reding, 2000) or $\geq 3$ (da Cunha et al., 2002; Sullivan et al., 2007) to various different indications on speed, endurance and assistance in walking, e.g. Da Cunha et al. 2002 (da Cunha et al., 2002) and Gama et al. 2017 (Gama et al., 2017). According to our findings, TT without any kind of support and with PT supervision was considered safe for FAC $>1$ and Tinetti values $>22$. Otherwise, BWSTT was guaranteed to all patients on a 1:1 PT/patient ratio with $\mathrm{FAC} \leq 1$ and Tinetti $\leq 5$. These indications need to be confirmed in a prospective study verifying the absence of adverse events like falls, which are often described in post-stroke patients, although with a low frequency (Mehrholz et al., 2017). Some examples occurred in the analyzed cases. Of note, there were four cases in which the algorithm (highlighting its attention to patients' security) indicated a safer modality of TT than that of the initial clinical decision. In these cases the initial decision was changed in progress and the four patients switched to a different modality of TT reflecting the algorithm's RS class.

Functional capacities (such as walking speed or endurance training) and psychological status are difficult to detail with a simple algorithm for post-stroke patients. Walking speed is considered a significant, sensitive and reliable indicator of the severity of the deficit and of the functional ability to walk in community (Bijleveld-Uitman, van de Port, \& Kwakkel, 2013; van de Port, Kwakkel, \& Lindeman, 2008), but it is applicable only to post-stroke patients with good functional skills (Fulk, Reynolds, Mondal, \& Deutsch, 2010). However, patients can walk quickly using compensatory strategies, so gait quality should also be considered (van de Port et al., 2008). Endurance is also an important parameter in assessing the severity of the deficit and the functional ability to walk (Bijleveld-Uitman et al., 2013; van de Port et al., 2008) but one must bear in mind that these patients are often deconditioned (An, Lee, Shin, \& Lee, 2015; Pang et al., 2013). It is also necessary to consider that these patients may experience anxiety on the treadmill and that walking on a treadmill with or without body weight support is not equivalent to 
walking on the ground, due to a different biomechanical activation (An et al., 2015). For all these reasons the physical therapist's holistic expertise could play an important role in "fine-tuning" the risk class.

The scope of our study was not to add new information on "what and how" to perform TT, but rather to provide an objective, standardized and repeatable way for health professionals to choose the best means of TT. In fact, the algorithm should create a guided decision making process that any PT can follow in order to choose the most appropriate TT mode for a given patient. However, the final responsibility of the decision of "if and how", eventually rejecting the indications provided by the algorithm, remains an issue for discussion and agreement by health professionals. For example, the PT can decide to include in RS4, the class of maximum safety (BWS with a dedicated physiotherapist), a specific patient not indicated as such by the algorithm, e.g. on the grounds of clinical criteria. This decision is, of course, at the discretion of the clinician/physiotherapist.

The decision making algorithm is not limited to a simple management of patient's training but can serve as a guide for making further objective choices, e.g. regarding the amount of PT time needed to dedicate to a patient (i.e. 1:1 PT/patient ratio, or PT supervision only allowing the PT to follow several patients at the same time) or the need for support bars during TT supporting a best use of the human resources available.

\subsection{Limitations}

The single recruiting center and the low number of patients in this study may constitute limitations. The retrospective study design forced the studied measured variables to the ones routinely evaluated, excluding other important variables (e.g. ICF core set, Fugle Meyer, Berg Balance, 6 minutes walking test). Moreover, the fact that there were 3 times more patients in the FreeTT group compared to the BSWTT group may have affected the statistics.

\subsection{Clinical and translational implications}

The algorithm developed in this study, despite its retrospective nature limited to a single cohort of patients and to a specific training system in neurological rehabilitation field, could eventually be extended, with appropriate adaptations, to: a) other neurological diseases (i.e. Parkinson's disease, other neuropathies)

b) other rehabilitation areas (i.e. cardiac and pulmonary diseases) in order to make the algorithm a function-dependent (i.e. walking) rather than a disease-dependent instrument.

c) other training tools (i.e. balance board, cycle ergometer).

Future prospective studies are warranted to evaluate the feasibility of the algorithm (in terms of the available resources and absence of adverse events), its efficacy (comparing improvement of patients' walking ability between the different RS classes) and the related costs and volumes of the services offered.

\section{Conclusions}

The elaboration of this decision making algorithm provides a guide for allocating post-stroke patients to TT on admission to the rehabilitation facility. The tool allows to objectively select the most appropriate mode of TT (free TT or with BWS, with a dedicated PT or under PT supervision only). Use of the algorithm can be extremely useful in the organizational context of an operative unit such as a rehabilitation gym, as it helps to make objective and repeatable decisions regarding the feasibility and safety of specific rehabilitation practices and to make best use of the human and instrumental resources available.

\section{Acknowledgments}

The Authors thank Rosemary Allpress for the English revision of the manuscript, Gualtiero Altieri and the PT team who treated the patients evaluated in this study

\section{Author contributions}

$\mathrm{FV}$ and $\mathrm{AO}$ equally contributed to the manuscript. FV conceived the idea, designed the study, performed literature search, collected data, and drafted the manuscript. AO designed the study, performed data curation, data visualization and statistical analysis, and drafted the manuscript. GPB, SD and MG collected data and GPB, SD, MG and LC critically reviewed the manuscript. LC and AL made a substantial contribution as supervisors and reviewers for 
the final manuscript version. All the authors critically reviewed the final version and approved the manuscript.

\section{Conflict of interest}

The authors have no conflict of interest to disclose related to the manuscript.

\section{Funding}

This work was supported by the "Ricerca Corrente" Funding scheme of the Ministry of Health, Italy.

\section{Supplementary data}

The supplementary files are available from https://dx.doi.org/10.3233/NRE-210065.

\section{References}

Ada, L., Dean, C. M., Hall, J. M., Bampton, J., \& Crompton, S. (2003). A treadmill and overground walking program improves walking in persons residing in the community after stroke: a placebo-controlled, randomized trial. Arch Phys Med Rehabil, 84(10), 1486-1491. doi:10.1016/s0003-9993(03)00349-6

Ada, L., Dean, C. M., \& Lindley, R. (2013). Randomized trial of treadmill training to improve walking in community-dwelling people after stroke: the AMBULATE trial. Int J Stroke, 8(6), 436-444. doi:10.1111/j.1747-4949.2012.00934.x

Ada, L., Dean, C. M., Morris, M. E., Simpson, J. M., \& Katrak, P. (2010). Randomized trial of treadmill walking with body weight support to establish walking in subacute stroke: the MOBILISE trial. Stroke, 41(6), 1237-1242. doi:10.1161/strokeaha.109.569483

An, S., Lee, Y., Shin, H., \& Lee, G. (2015). Gait velocity and walking distance to predict community walking after stroke. Nurs Health Sci, 17(4), 533-538. doi:10.1111/nhs. 12234

Barbeau, H., \& Visintin, M. (2003). Optimal outcomes obtained with body-weight support combined with treadmill training in stroke subjects. Arch Phys Med Rehabil, 84(10), 1458-1465. doi:10.1016/s0003-9993(03)00361-7

Bijleveld-Uitman, M., van de Port, I., \& Kwakkel, G. (2013). Is gait speed or walking distance a better predictor for community walking after stroke? J Rehabil Med, 45(6), 535-540. doi:10.2340/16501977-1147

Bohannon, R. W., Andrews, A. W., \& Smith, M. B. (1988). Rehabilitation goals of patients with hemiplegia. Int J Rehabil Res, 11(2), 181-183.

Bonnyaud, C., Pradon, D., Zory, R., Bussel, B., Bensmail, D., Vuillerme, N., \& Roche, N. (2013). Effects of a gait training session combined with a mass on the non-paretic lower limb on locomotion of hemiparetic patients: a randomized controlled clinical trial. Gait Posture, 37(4), 627-630. doi:10.1016/j.gaitpost.2012.09.010

Brauer, S. G., Kuys, S. S., Paratz, J. D., \& Ada, L. (2018). Improving physical activity after stroke via treadmill training and self management (IMPACT): a protocol for a randomised controlled trial. BMC Neurol, 18(1), 13. doi:10.1186/s12883018-1015-6

Casaubon, L. K., Boulanger, J. M., Glasser, E., Blacquiere, D., Boucher, S., Brown, K.,... Lindsay, P. (2016). Canadian Stroke Best Practice Recommendations: Acute Inpatient Stroke Care Guidelines, Update 2015. Int J Stroke, 11(2), 239252. doi:10.1177/1747493015622461

da Cunha, I. T., Jr., Lim, P. A., Qureshy, H., Henson, H., Monga, T., \& Protas, E. J. (2002). Gait outcomes after acute stroke rehabilitation with supported treadmill ambulation training: a randomized controlled pilot study. Arch Phys Med Rehabil, 83(9), 1258-1265. doi:10.1053/apmr.2002.34267

Dickstein, R. (2008). Rehabilitation of gait speed after stroke: a critical review of intervention approaches. Neurorehabil Neural Repair, 22(6), 649-660. doi:10.1177/15459 683080220060201

Dionisio, A., Duarte, I. C., Patricio, M., \& Castelo-Branco, M. (2018). The Use of Repetitive Transcranial Magnetic Stimulation for Stroke Rehabilitation: A Systematic Review. J Stroke Cerebrovasc Dis, 27(1), 1-31. doi:10.1016/ j.jstrokecerebrovasdis.2017.09.008

Eng, J. J., \& Tang, P. F. (2007). Gait training strategies to optimize walking ability in people with stroke: a synthesis of the evidence. Expert Rev Neurother, 7(10), 1417-1436. doi:10.1586/14737175.7.10.1417

Esquenazi, A., Lee, S., Wikoff, A., Packel, A., Toczylowski, T., \& Feeley, J. (2017). A Comparison of Locomotor Therapy Interventions: Partial-Body Weight-Supported Treadmill, Lokomat, and G-EO Training in People With Traumatic Brain Injury. Pm $r, 9(9), 839-846$. doi:10.1016/j.pmrj.2016.12.010

Franceschini, M., Carda, S., Agosti, M., Antenucci, R., Malgrati, D., \& Cisari, C. (2009). Walking after stroke: what does treadmill training with body weight support add to overground gait training in patients early after stroke?: a singleblind, randomized, controlled trial. Stroke, 40(9), 3079-3085. doi:10.1161/strokeaha.109.555540

Fulk, G. D., Reynolds, C., Mondal, S., \& Deutsch, J. E. (2010). Predicting home and community walking activity in people with stroke. Arch Phys Med Rehabil, 91(10), 1582-1586. doi:10.1016/j.apmr.2010.07.005

Gama, G. L., Celestino, M. L., Barela, J. A., Forrester, L., Whitall, J., \& Barela, A. M. (2017). Effects of Gait Training With Body Weight Support on a Treadmill Versus Overground in Individuals With Stroke. Arch Phys Med Rehabil, 98(4), 738-745. doi:10.1016/j.apmr.2016.11.022

Globas, C., Becker, C., Cerny, J., Lam, J. M., Lindemann, U., Forrester, L. W.,... Luft, A. R. (2012). Chronic stroke survivors benefit from high-intensity aerobic treadmill exercise: a randomized control trial. Neurorehabil Neural Repair, 26(1), 85-95. doi:10.1177/1545968311418675

Granger, C. V., Cotter, A. C., Hamilton, B. B., \& Fiedler, R. C. (1993). Functional assessment scales: a study of persons after stroke. Arch Phys Med Rehabil, 74(2), 133-138.

Gresham, G. E., Phillips, T. F., Wolf, P. A., McNamara, P. M., Kannel, W. B., \& Dawber, T. R. (1979). Epidemiologic profile of long-term stroke disability: the Framingham study. Arch Phys Med Rehabil, 60(11), 487-491. 
Holden, M. K., Gill, K. M., \& Magliozzi, M. R. (1986). Gait assessment for neurologically impaired patients. Standards for outcome assessment. Phys Ther, 66(10), 1530-1539. doi:10.1093/ptj/66.10.1530

Istruzione operativa ICS Maugeri Gestione delle cadute accidentali (IO ICSM CGA rev0 05/12/2018). (2018). In.

Kim, C. S., Gong, W., \& Kim, S. G. (2011). The Effects of Lower Extremitiy Muscle Strengthening Exercise and Treadmill Walking Exercise on the Gait and Balance of Stroke Patients. Journal of Physical Therapy Science, 23(3), 405-408. doi: $10.1589 /$ jpts.23.405

Kosak, M. C., \& Reding, M. J. (2000). Comparison of partial body weight-supported treadmill gait training versus aggressive bracing assisted walking post stroke. Neurorehabil Neural Repair, 14(1), 13-19. doi:10.1177/154596830001400102

Luft, A. R., Macko, R. F., Forrester, L. W., Villagra, F., Ivey, F., Sorkin, J. D.,... Hanley, D. F. (2008). Treadmill exercise activates subcortical neural networks and improves walking after stroke: a randomized controlled trial. Stroke, 39(12), 33413350. doi:10.1161/strokeaha.108.527531

Mao, Y. R., Lo, W. L., Lin, Q., Li, L., Xiao, X., Raghavan, P., \& Huang, D. F. (2015). The Effect of Body Weight Support Treadmill Training on Gait Recovery, Proximal Lower Limb Motor Pattern, and Balance in Patients with Subacute Stroke. Biomed Res Int, 2015, 175719. doi:10.1155/2015/175719

Mehrholz, J., Thomas, S., \& Elsner, B. (2017). Treadmill training and body weight support for walking after stroke. Cochrane Database Syst Rev, 8, Cd002840. doi:10.1002/14651858.CD002840.pub4

Morse, J. M., Morse, R. M., \& Tylko, S. J. (1989). Development of a Scale to Identify the Fall-Prone Patient. Canadian Journal on Aging / La Revue canadienne du vieillissement, 8(4), 366-377. doi:10.1017/S0714980800008576

Ng, M. F., Tong, R. K., \& Li, L. S. (2008). A pilot study of randomized clinical controlled trial of gait training in subacute stroke patients with partial body-weight support electromechanical gait trainer and functional electrical stimulation: six-month follow-up. Stroke, 39(1), 154-160. doi:10.1161/strokeaha.107.495705

Oliver, D., Britton, M., Seed, P., Martin, F. C., \& Hopper, A. H. (1997). Development and evaluation of evidence based risk assessment tool (STRATIFY) to predict which elderly inpatients will fall: case-control and cohort studies. Bmj, 315(7115), 1049-1053. doi:10.1136/bmj.315.7115.1049

Pang, M. Y., Charlesworth, S. A., Lau, R. W., \& Chung, R. C. (2013). Using aerobic exercise to improve health outcomes and quality of life in stroke: evidence-based exercise prescription recommendations. Cerebrovasc Dis, 35(1), 7-22. doi: $10.1159 / 000346075$

Parmelee, P. A., Thuras, P. D., Katz, I. R., \& Lawton, M. P. (1995). Validation of the Cumulative Illness Rating Scale in a geriatric residential population. J Am Geriatr Soc, 43(2), 130-137.

Rensink, M., Schuurmans, M., Lindeman, E., \& Hafsteinsdottir, T. (2009). Task-oriented training in rehabilitation after stroke: systematic review. $J$ Adv Nurs, 65(4), 737-754. doi:10.1111/j.1365-2648.2008.04925.x

Selves, C., Stoquart, G., \& Lejeune, T. (2020). Gait rehabilitation after stroke: review of the evidence of predictors, clinical outcomes and timing for interventions. Acta Neurol Belg, 120(4), 783-790. doi:10.1007/s13760-020-01320-7

Sullivan, K. J., Brown, D. A., Klassen, T., Mulroy, S., Ge, T., Azen, S. P., \& Winstein, C. J. (2007). Effects of task-specific locomotor and strength training in adults who were ambulatory after stroke: results of the STEPS randomized clinical trial. Phys Ther, 87(12), 1580-1602. doi:10.2522/ptj.20060310

Tinetti, M. E. (1986). Performance-oriented assessment of mobility problems in elderly patients. J Am Geriatr Soc, 34(2), 119-126. doi:10.1111/j.1532-5415.1986.tb05480.x

van de Port, I. G., Kwakkel, G., \& Lindeman, E. (2008). Community ambulation in patients with chronic stroke: how is it related to gait speed? J Rehabil Med, 40(1), 23-27. doi:10.2340/16501977-0114

White Book on Physical and Rehabilitation Medicine in Europe. Introductions, Executive Summary, and Methodology. (2018). Eur J Phys Rehabil Med, 54(2), 125-155. doi:10.23736/s19739087.18.05143-2

Winstein, C. J., Stein, J., Arena, R., Bates, B., Cherney, L. R., Cramer, S. C.,... Zorowitz, R. D. (2016). Guidelines for Adult Stroke Rehabilitation and Recovery: A Guideline for Healthcare Professionals From the American Heart Association/American Stroke Association. Stroke, 47(6), e98-e169. doi:10.1161/str.0000000000000098 